\title{
Accelerated hydrotherapy and land-based rehabilitation in soccer players after anterior cruciate ligament reconstruc- tion: a series of three single subject case studies
}

\author{
Bridey-Lee Momberg (BSc, MSc Physiotherapy (OMT)) \\ Quinette Louw (BSc, MASP, PhD) \\ Lynette Crous (BSc, MSc)
}

Division of Physiotherapy, University of Stellenbosch

\begin{abstract}
Objective. To investigate the effectiveness of accelerated rehabilitation and accelerated hydrotherapy after anterior cruciate ligament $(A C L)$ reconstruction in male athletes participating in soccer.

Design. A non-concurrent single subject, multiple baseline design (ABA design) was conducted over 10 weeks. A series of three $\mathrm{N}=1$ studies was conducted to assess the effect of an accelerated hydrotherapy programme on pain, function, and range of motion.

Setting. The study was conducted at a private physiotherapy practice in Port Elizabeth, South Africa.

Interventions. The land rehabilitation programme was a homebased programme supervised every week by the physiotherapist. The accelerated hydrotherapy consisted of a 6-week programme, and participants attended two treatment sessions of accelerated hydrotherapy per week each of 30 minutes' duration.

Main outcome measures. The knee injury and osteoarthritis outcome scale (KOOS) as a subjective measure of pain, function and quality of life; the goniometer to measure active knee ROM and the 6-minute walking test (6MWT) as an objective measure of function.
\end{abstract}

Results. All three patients demonstrated good improvement during the treatment phase for the KOOS scale and progressed well

\section{CORRESPONDENCE:}

Professor Quinette Louw

Division of Physiotherapy

Department of Interdisciplinary Health Sciences

Faculty of Health Sciences

Stellenbosch University

PO Box 19063

Tygerberg 7505

Republic of South Africa

Tel: +27(021) 938 - 9301

Fax: +27(021) 931-1252

E-mail: qalouw@sun.ac.za in terms of their walking ability during the study. Significant improvement was gained during the baseline phase for all three participants with high initial levels of knee flexion while active knee extension improved gradually in all three participants.

Conclusion. The study findings indicate that an accelerated landbased and hydrotherapy programme may be useful in improving patient outcomes and that there are no risks for harm.

Clinical relevance. The study findings indicate that accelerated hydrotherapy may be a useful and safe adjunct to an accelerated land-based programme after ACL reconstruction.

\section{Introduction}

Hydrotherapy is an excellent training medium for rehabilitation in the athlete who is under time constraints for recovery after a sporting injury. ${ }^{20}$ Aquatic exercises have been recommended, especially in the initial phase of rehabilitation to allow early active mobilisation and to improve neuromuscular function. ${ }^{14}$

The primary goals of accelerated rehabilitation following an ACL reconstruction are to recover joint range of motion (ROM), strength, ambulatory skills and return to previous activity or sport. ${ }^{18,21}$ Hydrotherapy may provide the ideal medium to accomplish these goals due to the positive effects of the physical properties of water on the human body, such as decreased pain, increased ROM and flexibility, early restoration of joint mobility, reduced oedema and increased blood flow to muscles. ${ }^{2,6,14,16,20}$

Published research into the physical properties and biomechanical effects of water on the knee provides support for the use of hydrotherapy in knee rehabilitation. 2,14,15,16,20 Biomechanical studies demonstrated that accelerated open (OKC) and closed kinematic chain $(\mathrm{CKC})$ exercises in water are not detrimental to the healing ACL graft. ${ }^{2,14,15}$ In water, the knee joint plays no role in the absorption of forces because the impact force is greatly reduced due to buoyancy. ${ }^{11,12}$ This provides an opportunity to accomplish early full weight-bearing in water to enable gait re-education.

Hydrotherapy may thus increase a patient's tolerance to rehabilitation programmes. Although accelerated land-based programmes have been published extensively, a combination of accelerated land and hydrotherapy programmes has not been studied. A combined programme may be beneficial to sports participants as it allows more joint loading, aggressive rehabilitation and earlier return to function. ${ }^{21}$ 
The aim of this study was to investigate the effectiveness of the addition of accelerated hydrotherapy to standard accelerated land-based rehabilitation in reducing pain and increasing ROM and functional outcomes after ACL reconstruction in male athletes participating in soccer.

\section{Methodology}

A series of three $\mathrm{N}=1$ studies was conducted to assess the effect of an accelerated hydrotherapy programme on pain, function and ROM.

The sample comprised participants who fulfilled the following inclusion criteria: they were English-proficient male competitive soccer players (aged 20 - 44 years) who had suffered a traumatic ACL injury while participating in soccer, who subsequently underwent unilateral left or right $A C L$ repairs (irrespective of dominance) and who suffered a complete ACL rupture with or without an associated meniscal injury. Participants were included in the study if they participated in soccer at a level where they played matches for a recognised team and participated in a league or tournament. The surgery was performed by the same surgeon who used the arthroscopic four-strand hamstring (semitendinosis and gracilis) single bundle graft method to repair the ACL. Participants were excluded from the study if they: fell outside the described age group, suffered an ACL injury through another mechanism of injury other than soccer, were diagnosed with multidirectional instability of the affected knee, suffered any acute injury to the contralateral lower limb or had associated ankle and hip injuries on the ipsilateral side.

Ethics approval was obtained from the Committee for Human Research at the Stellenbosch University (project number: N06/10/207) and all subjects provided signed consent.

\section{Study design}

A non-concurrent single subject, multiple baseline design (ABA design) was conducted over 10 weeks. Phase A1 represented the baseline phase, commenced at day 10 post-surgery, and consisted of an accelerated land rehabilitation programme. Phase B represented the treatment phase and consisted of both land rehabilitation and accelerated hydrotherapy. The duration of phase B was 6 weeks. The three participants entered the treatment phase at different times in their post-surgical rehabilitation and this was allocated in a consecutive manner as presented in Table I. Phase A2 was the withdrawal phase, where the accelerated hydrotherapy was withdrawn and the accelerated land rehabilitation programme continued until 12 weeks after surgery (Table I).

The study was conducted at a private physiotherapy practice in Port Elizabeth, South Africa. Participants were recruited from the practice of an orthopaedic surgeon who had agreed to refer eligible patients. Participants were referred once the surgeon was satisfied that structural stability was satisfactory after the surgery. A consecutive sampling method was used. Since there was no order in which patients presented to the surgeon's office, arguable random participants were recruited.

\section{Outcome measurement}

Three baseline measures for each outcome were measured before the participant entered the treatment phase. During the treatment phase six measurements for each outcome were recorded (these were taken before the treatment was administered) and during the withdrawal phase another three measures for each outcome were taken (Table II).

\section{Reliability and validity of the outcome measures}

The intra- and inter-tester reliability in the study by Brosseau et al. was high for the universal goniometer with high intraclass correlation coefficients (ICCs) for both flexion $(R=0.99)$ and extension $(R=0.9$ 0.98 ) for the knee. ${ }^{3}$ The validity was studied using radiographs as the gold standard. ${ }^{3}$ Validity was high for measuring knee flexion $(R=0.97$ $0.98)$, but lower for measuring knee extension $(R=0.39-0.44){ }^{3}$

KOOS is a knee-specific instrument which has 42 items in 5 separately scored sub-scales: pain, other symptoms, function in daily living (ADL), function in sport and recreation (sport/rec), and knee-related quality of life (QOL). ${ }^{17}$ KOOS has been validated in participants undergoing $A C L$ reconstruction. ${ }^{17}$

The 6-minute walking test (6MWT) is a time-modified test that originated from the original 12-minute walking test as a field test for assessing maximal oxygen uptake in athletes. Kennedy et al. assessed the reliability and sensitivity to change of the 6MWT in hip and knee osteoarthritis (OA) sufferers with good results. ${ }^{8}$ In the study by Kennedy et al. the ICC for the 6MWT was $\mathrm{R}=0.94(0.88,0.98){ }^{8}$

\section{Study procedures}

Each participant received a patient booklet (containing the consent form, information about the respective practices, summary of the rehabilitation programmes and exercise descriptions with appropriate diagrams) and a diary of compliance in which they were asked to record their compliance with the accelerated land rehabilitation programme.

All participants received preoperative rehabilitation. This was a 6-week land-based programme and was administered by the researching physiotherapist. Post-surgical management in hospital was standardised and consisted of circulatory exercises and mobilisation (partial weightbearing on two crutches) conducted by the researcher. For the first 10 days a standard rehabilitation protocol was followed (Table III). The study started on day 10 post-surgery. A

\begin{tabular}{|c|c|c|c|c|c|c|c|c|c|c|c|}
\hline $\mathbf{P t}$ & D10-14 & W 2-3 & W 3-4 & W 4-5 & W 5-6 & W 6-7 & W 7-8 & W 8-9 & W 9-10 & W 10-11 & W11-12 \\
\hline 1 & ACR & $H \& A C R$ & $\mathrm{H} \& \mathrm{ACR}$ & $\mathrm{H} \& \mathrm{ACR}$ & $\mathrm{H} \& \mathrm{ACR}$ & $\mathrm{H} \& \mathrm{ACR}$ & $\mathrm{H} \& \mathrm{ACR}$ & ACR & ACR & ACR & ACR \\
\hline 2 & ACR & ACR & $\mathrm{H} \& \mathrm{ACR}$ & $H \& A C R$ & $H \& A C R$ & $H \& A C R$ & $H \& A C R$ & $\mathrm{H} \& \mathrm{ACR}$ & ACR & ACR & ACR \\
\hline 3 & ACR & ACR & ACR & $\mathrm{H} \& \mathrm{ACR}$ & $\mathrm{H} \& \mathrm{ACR}$ & $\mathrm{H} \& \mathrm{ACR}$ & $H \& A C R$ & $H \& A C R$ & $H \& A C R$ & ACR & ACR \\
\hline \multicolumn{12}{|c|}{$\mathrm{D}=$ days post-surgery; $\mathrm{W}=$ weeks post-surgery; $\mathrm{ACR}=$ accelerated land rehabilitation programme; $\mathrm{H} \& \mathrm{ACR}=$ accelerated hydrotherapy and accelerated land rehabilitation programme. } \\
\hline \multicolumn{12}{|c|}{$\mathrm{A} 1=$ baseline (ACR only) } \\
\hline \multicolumn{12}{|c|}{$\square \mathrm{B}=$ treatment phase (ACR and accelerated hydrotherapy) } \\
\hline \multicolumn{12}{|c|}{ A2 $=$ withdrawal phase (ACR only, accelerated hydrotherapy was withdrawn) } \\
\hline
\end{tabular}




\section{TABLE II. Outcome measurement}

\begin{tabular}{llll}
\hline Phase & A1 (Baseline) & B (Treatment) & A2 (Withdrawal) \\
KOOS & 3 measures & 6 measures & 3 measures \\
Goniometer & 3 measures & 6 measures & 3 measures \\
6 MWT & 3 measures & 6 measures & 3 measures \\
Participant 1 & D10-14 & W2-8 & W8-12 \\
Participant 2 & D10-W3 & W3-9 & W9-12 \\
Participant 3 & D10-W4 & W4-10 & W10-12 \\
KOOS = subjective pain, function and QOL; goniometer = active ROM; 6-minute walking test (6MWT) = function.
\end{tabular}

\section{TABLE III. Initial post-surgical treatment (day 1 - 10) (MacDonald et al., ${ }^{10}$ Tovin et al. ${ }^{21}$ )}

\section{Goal}

Improve circulation

Restore range of motion (no restrictions except outside pain)

Improve strength

Reduce swelling

\section{Treatment}

Circulatory exercises

Assisted extension and flexion outside pain (25X), wall slides (25X), passive knee extension roll under ankle or prone hangs(10 minutes), hamstring and calf stretching (30 seconds, $3 \mathrm{X} /$ day)

Isometric quadriceps (3X10)

SLR with hip flexion and hip abduction (3X10 each)

FWB on 2 crutches

Cryotherapy, RICE regimen

$\mathrm{SLR}=$ straight leg raise; FWB = full weight bearing; RICE = rest, ice, compression and elevation.

subjective and physical examination was conducted according to the principles of Maitland at commencement of the baseline phase. ${ }^{7}$

A pilot study was done to determine the intertester and intratester reliability and to standardise the goniometry procedures. This was executed in the baseline phase and the measures were taken by the principal investigator and a second physiotherapist. Each physiotherapist took two measures of the goniometer measurements on a given day. This was repeated on three separate occasions during the baseline phase for each of the participants.

\section{Description of the intervention}

Throughout the 10-week study the participants followed the accelerated land rehabilitation programme, beginning day 10 post-surgery until completion of the study at 12 weeks post-surgery. The land rehabilitation programme was a home-based programme supervised every week by the physiotherapist (Table IV). The patient booklet provided a detailed description of each exercise and the compliance diary was kept. The land programme focussed on restoration of muscle strength, proprioception, functional strengthening and return to sport. 4

Each of the three participants then entered the treatment phase at 2,3 or 4 weeks post-surgery respectively. This consisted of a 6 -week accelerated hydrotherapy programme (Table V). The intervention of hydrotherapy was implemented at different times with the goal of observing a noticeable change with its implementation. Thus this change could then be attributed to the addition of hydrotherapy if it occurred in each participant and not only due to post-surgical maturation.

During the hydrotherapy phase, the participants attended two treatment sessions of accelerated hydrotherapy per week, each of 30 minutes' duration, for the 6-week intervention period. They then ceased the additional hydrotherapy programme (at 8,9 or 10 weeks in the study period respectively) and continued the land-based rehabilitation only for the withdrawal (A2) period. At 12 weeks the participants had their final assessment and the formal part of the study was completed. They then continued with the accelerated land rehabilitation programme, and were monitored and progressed on a monthly basis until 6 months after surgery, when they were referred for further sports-specific rehabilitation if necessary, until such time as they had returned to their sport. All outcomes were measured by the researcher. During the accelerated hydrotherapy phase, all outcomes were measured before the hydrotherapy session. Further details of the interventions are available from the authors.

\section{Data analysis}

All data were entered into Microsoft Excel for analysis. Descriptive statistics were used to summarise the data. Visual analysis of graphs and effect size were calculated to analyse performance. ${ }^{1}$ An effect size of 2.6 was classified as small, 3.9 as moderate and 5.8 and higher a large effect. ${ }^{19}$ Intra-class correlations (ICC) using the two-way model were calculated using the statistical programming language $\mathrm{R}$. The ICCs were calculated to determine the reliability of the knee ROM measurements.

\section{Results}

There was excellent intra- and inter-tester reliability for knee flexion and knee extension in the pilot study. The ICC values for knee flexion were 0.99 and 0.91-0.95 for knee extension intra- and inter-tester reliability.

\section{Participant descriptions}

Participant 1 was a 26-year-old male who sustained an injury to his left knee 3 months before his scheduled ACL reconstruction. The mechanism of injury was a twisting action of the knee on a fixed foot. He experienced immediate and continued pain and swelling after his injury. 
TABLE IV. Accelerated land-based rehabilitation programme (Fujimoto et al.. ${ }^{5}$ MacDonald et al. ${ }^{10}$, Muneta et al. ${ }^{13}$ )

\begin{tabular}{ll}
\hline $\begin{array}{l}\text { Week } \\
0-2 \text { weeks } \\
2 \text { weeks }\end{array}$ & Criteria met \\
4 weeks & Flexion to 90 \\
& \\
& \\
& Full ROM, no quadriceps lag, no \\
& limping and able to do SLR
\end{tabular}

8 weeks limping and able to do SLR

10 weeks

12 weeks

16 - 24 weeks

(4 - 6 months)

Full ROM and quadriceps muscle strength $85 \%$ of unoperated leg

24 - 32 weeks with isokinetic testing

Treatment

As post-surgical treatment (d 1-10)

Continue with exercises, cycling

Continue with exercises, cycling Strengthening exercisesCKC (leg presses and squats)

Hip strengthening against resistance $(F, E$, abduction and adduction)

Ankle strengthening in standing (calf raises)

Ambulation without crutches

Cycling

Hamstring strengthening (concentric and eccentric)

Continuation of hip and calf strengthening

Gait re-education

Jogging (straight-ahead, level ground, with no pivoting)

Continuing with other exercises

Jogging continued

Return to non-pivoting sports

Return to pivoting sports

TABLE V. Accelerated hydrotherapy programme (Biscarini and Cerulli, ${ }^{2}$ Kuehne and Zirkel, ${ }^{9}$ Miyoshi et al.., ${ }^{11,12}$ Poyhonen et al., ${ }^{14}$ Tovin et al. ${ }^{21}$ )

\section{Goal}

Gait retraining

Quadriceps and hamstring strengthening

Strengthening and endurance training

Hip and calf maintenance and strengthening

Balance and proprioception (perturbation protocol)

Plyometric training

Sports-specific rehabilitation

\author{
Treatment \\ Gait training \\ Closed kinetic chain exercises \\ Open kinematic chain exercises \\ Running \\ Cycling \\ Deep-water running techniques \\ Hip exercises and calf raises, kicking and vertical kicking \\ Balance and proprioception exercises \\ Jumping, running, shuttle runs, side steps with increased speed \\ Agility exercises making use of a ball
}

Participant 2 was a 28-year-old male who sustained an injury to his left knee 5 years ago. He underwent an arthroscopic debridement and returned to his activities of daily living. He then suffered a re-injury to his left knee 6 months before his scheduled ACL reconstruction. The mechanism of injury was a fast extension of the knee during a fall. He experienced immediate and continued pain and swelling with a significant disturbance in gait pattern.

Participant 3 was a 43-year-old male who sustained an injury to his right knee 8 months prior to his scheduled ACL reconstruction. The mechanism of injury was a twisting action of the knee on a fixed foot. Participant 3 experienced immediate pain and swelling, but after a period of rest had attempted to return to soccer. However, he experienced pain, swelling and giving way of his right knee.

\section{KoOS scale}

In all three participants, 18 - $28 \%$ of improvement in the KOOS scores was demonstrated in the baseline phase. All three patients demonstrated good improvement during the treatment and withdrawal phases. The effect size for the three participants was $8.00,8.66$ and 6.00 and indicated that a large effect was obtained during the intervention and withdrawal phases.

Measurement four is the transitional measure between the baseline and treatment phase and was taken just before the accelerated hydrotherapy was commenced. The difference between measures three and four was -1 (57.5 - 56.5) and between measures four and five was 9 (56.5 - 65.4), illustrating that participant 3's baseline had begun to stabilise and an increase in performance was noted after commencement of the accelerated hydrotherapy (Fig. 1).

\section{Objective functional ability 6MWT}

All three participants progressed well in terms of their walking ability. Participants 1 and 2 demonstrated between $16 \%$ and $23 \%$ of their overall improvement in walking ability in the baseline phase. Participant 3 , however, demonstrated $57 \%$ of his total improvement during the baseline phase.

The overall effect size (including treatment and withdrawal phases) for participant 1 was $4.38,6.49$ for participant 2 and 2.41 for participant 3. Participant 1 and 2 thus demonstrated relatively better performance during the treatment and withdrawal phases compared with participant 3 (Fig. 2).

\section{Active knee flexion ROM}

Fig. 3 illustrates that all three participants started at a relatively high initial level of knee flexion and much improvement was gained during the baseline phase. Thus the overall effect sizes (including treatment and withdrawal phases) were relatively smaller, 2.48 for participant 1, 3.50 for participant 2 and 1.79 for participant 3 , as they had almost reached full range at the commencement of the hydrotherapy. The remainder of the study all three participants continued to improve their active flexion ROM but at a slower rate, and measures began to plateau out towards the end of the study (Fig. 3). 


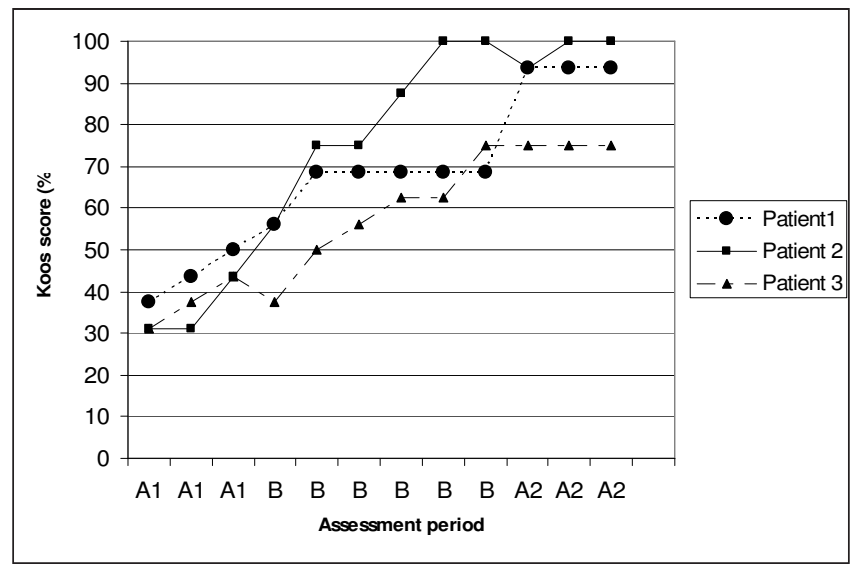

Fig. 1. KOOS score findings.

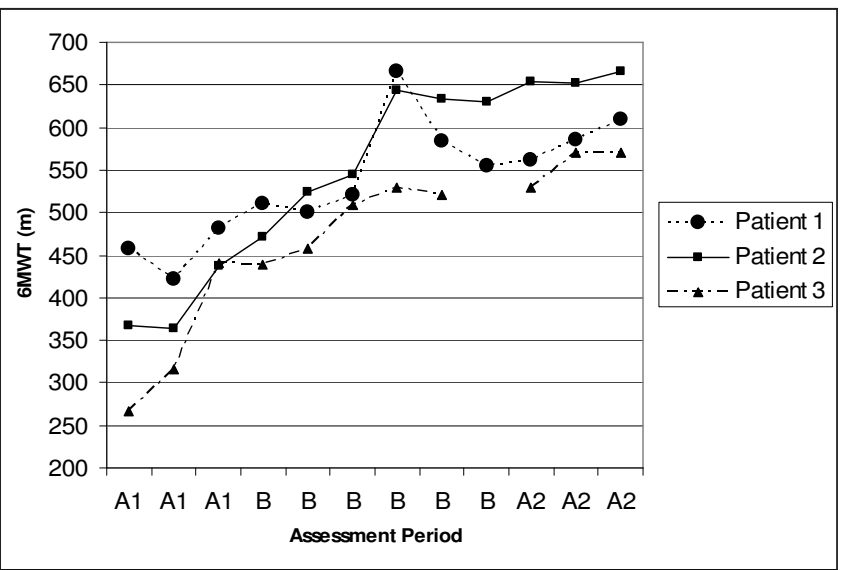

Fig. 2. 6MWT findings (participant 3 missed one session).

\section{Active knee extension ROM}

Active knee extension improved gradually in all three participants. At baseline, participant 1 had shown no change in his extension measure, participant 3 had shown a $45 \%$ improvement and participant 2 had $66 \%$ of his improvement occurring in the baseline phase.

Participant 1 demonstrated the largest effect (effect size 8.08) during the treatment and withdrawal phases compared with participant 2 (effect size 2.9) and participant 3 (2.66), although participant 2 obtained the most knee extension at the end of the study (Fig. 4).

\section{Discussion}

The study findings indicate that an accelerated hydrotherapy programme in addition to a land-based programme may be useful in improving patient outcomes and that there are no risks for harm. Care and awareness of the healing hamstring at the donor site must, however, be kept in mind throughout the rehabilitation programme. The physical properties of water result in biological effects on the body such as the reduction in pain, increases in ROM, improved coordination of movement and early restoration of joint ROM. ${ }^{2,6,14,16,20}$ These biological effects provide reasons why all the participants' perceptions of their pain, symptoms, function and QOL improved during the treatment phase with the addition of hydrotherapy. Experimental studies demonstrate that hydrotherapy has a positive effect on the reduction of pain and improvements in functional scales after ACL reconstruction. 9,21

Participant 3 showed a stabilising baseline at measures three and four and then a marked increase between measures four and

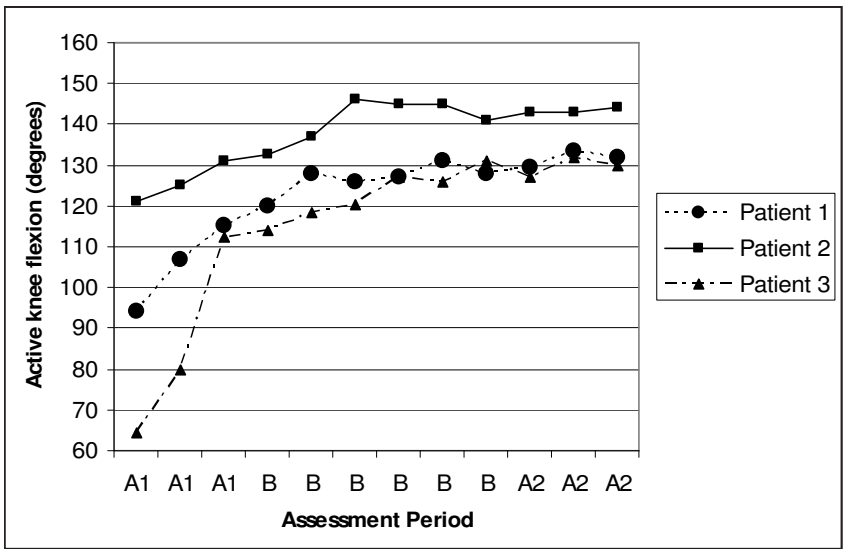

Fig. 3. Active knee flexion ROM findings.

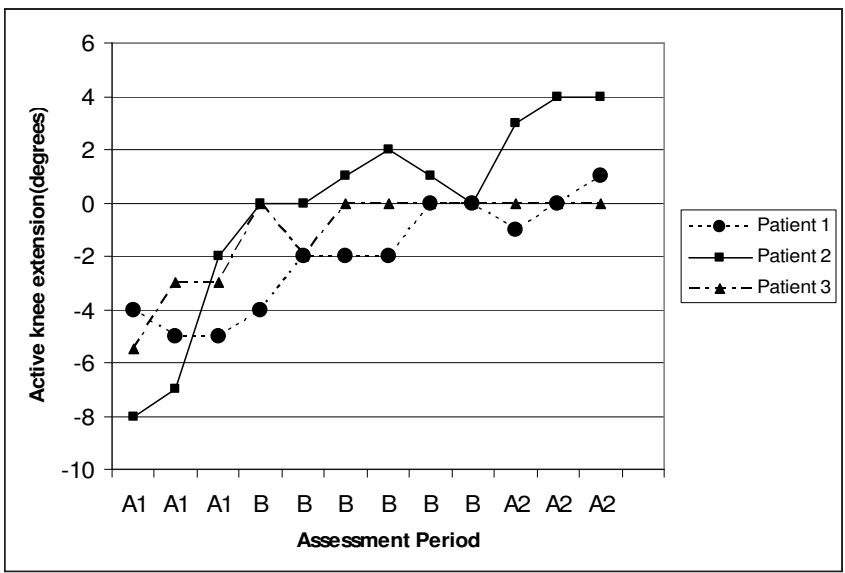

Fig. 4. Active knee extension ROM findings.

five for the KOOS score (Fig. 1). Hydrotherapy may therefore have improved participant 3's perception of his pain, symptoms, function and QOL. Participant 3 entered the treatment phase after a longer period compared with participants 1 and 2 and therefore his baseline measures may have stabilised and changes in performance after commencement of the hydrotherapy were more noticeable. The same change may not have been visible in the other two participants because of the unstable baseline phases shown in a number of KOOS measures for these participants; however, these two participants also demonstrated good effect of treatment on their KOOS scores.

All participants demonstrated steady improvements in their walking ability throughout the study. Participant 3 demonstrated relatively less effect during the treatment phase on walking ability. This may be because he started the hydrotherapy relatively later and had already regained significant improvement in walking ability before commencement of hydrotherapy. Maturation was therefore a threat to the internal validity, and the effect of this may be most notable in participant 3.

All participants obtained high initial knee flexion ROM even before the start of the study, therefore relatively small treatment effects were noted during the treatment phase. The participants' good knee flexion ROM may be due to accelerated post-surgical rehabilitation protocol of immediate full weightbearing and no bracing or restriction to movement, allowing earlier restoration of ROM before the start of the study and during baseline phases. The land-based rehabilitation protocol of emphasised knee extension immediately after surgery may explain why participants 2 and 3 obtained good extension before the start of the hydrotherapy. Participant 1 demonstrated the greatest 
effect of treatment on knee extension measures. This may, however, be better explained by post-surgical maturation as this participant had the shortest baseline phase and all three participants obtained between $50 \%$ and $100 \%$ of maximum range by 4 weeks after surgery (Fig. 4). Although the multiple baseline design was applied to control for internal validity threats such as maturation, the effects of maturation could not be completely negated.

\section{Study limitations}

The present study, due to its clinical nature, did not allow access to the KT-1000 (gold standard for determining graft stability) for objective determination of graft stability which is of obvious concern with the implementation of accelerated rehabilitation protocols after ACL reconstruction.

Due to time constraints, long-term follow up of 6 months could not be done, and baseline phases were also kept to a minimum time period ranging from 4 to 18 days. Long-term effects of accelerated hydrotherapy should therefore be investigated in future studies. Participant satisfaction (though not investigated in this study) can be a good predictor of recovery and should be considered in future studies to explore the use of accelerated hydrotherapy in this patient population.

\section{Clinical implications}

- Accelerated hydrotherapy in addition to land-based rehabilitation is safe, feasible and well tolerated by participants.

- There are some trends in the data to indicate that it has an extra beneficial effect on subjective measures of pain, function and QOL (measured by the KOOS scale) over and above land-based rehabilitation.

- Clinicians should use this intervention with clear outcome measures to monitor individual effects until such time as there is a stronger evidence base for its effectiveness in sports persons after ACL reconstruction.

- Further research into the use of hydrotherapy in this population, using larger sample sizes and higher-level methodology, is now warranted.

\section{Conclusion}

It is essential that new approaches to management be studied in smaller case series to understand responses in outcomes prior to expensive, large-group intervention studies. This study's findings indicate that accelerated hydrotherapy may be a useful and safe adjunct to an accelerated land-based programme after ACL reconstruction. This study therefore provides an impetus for larger future experimental studies.

\section{Acknowledgements}

Dr VP Gajjar (orthopaedic surgeon) is acknowledged for his participation and contribution to this study.

\section{References}

1. Beeson PM, Robey RR. Evaluating single-subject treatment research: Lessons from Aphasia Literature. Neuropsychol Rev 2006; 16: 161-9.

2. Biscarini A, Cerulli $G$. Modelling of the knee joint load in rehabilitative knee extension exercises under water. J Biomech 2007; 40(2): 345-55.

3. Brosseau L, Balmer S, Tousignant M, et al. Intra- and inter-tester reliability and criterion Validity of the parallelogram and universal goniometers for measuring maximum active knee flexion and extension of patients with knee restrictions. Arch Phys Med Rehabil 2001; 82: 396-402.

4. Chmielewski TL, Hurd WJ, Rudolph KS, Axe MJ, Snyder-Mackler L. Perturbation training improves knee kinematics and reduces muscle co-contraction after complete unilateral anterior cruciate ligament rupture. Phys Ther 2005; 85(8): 740-9.

5. Fujimoto $E$, Sumen $Y$, Urabe $Y$, et al. An early return to vigorous activity may destabilise anterior cruciate ligament reconstructed with hamstring grafts. Arch Phys Med Rehabil 2004; 85(2): 298-302.

6. Heller L, Martin K. WTA Tour: aquatic therapy for tennis. Med Sci Tennis 2003; 8(1): 8-9.

7. Hengeveld E, Banks K. Maitland's Peripheral Manipulation, 4th ed. London: Butterworth Heinemann, 2005: 89-124.

8. Kennedy DM, Stratford PW, Wessel J, Gollish JD, Penney D. Assessing stability and change for four performance measures: a longitudinal study-evaluating outcome following total hip and knee arthroplasty. BMC Musculoskelet Disord 2005; 6(1): 3.

9. Kuehne C, Zirkel A. Accelerated rehabilitation following patellar tendon autograft anterior cruciate ligament reconstruction using the aqua-jogging protocol: a primary study. Sports Exerc Injur (Edinburgh, Scotland) 1996; 2(1): 15-23.

10. MacDonald PB, Hedden D, Pacin O, Huebert D. Effects of an accelerated rehabilitation program after anterior cruciate ligament reconstruction with combined semitendinosus-gracilis autograft and a ligament augmentation device. Am J Sports Med 1995; 23(5): 588-592.

11. Miyoshi T, Shiroto T, Yamamoto S-I, Nakazawa K, Akai M. Functional roles of lower limb joint moments while walking in water. Clin Biomech (Bristol Avon) 2005; 20(2): 194-201.

12. Miyoshi T, Shiroto T, Yamamoto S-I, Nakazawa K, Akai M. Lower limb joint moment during walking in water. Disabil Rehabil 2003; 25(21): 1219-23.

13. Muneta T, Sekiya I, Ogiuchi T, Yagishita K, Yamamoto H, Shimomiya K. Effects of aggressive early rehabilitation on the outcome of anterior cruciate ligament reconstruction with multistrand semitendinosus tendon. Int Orthop 1998; 22: 352-6.

14. Poyhonen T, Kyrolainen H, Keskinen KL, Hautala A, Savolainen J, Malkia E. Electromyographic and kinematic analysis of therapeutic knee exercises under water. Clin Biomech (Bristol Avon) 2001; 16: 496-504.

15. Poyhonen T, Keskinen KL, Kyrolainen H, Hautala A, Savolainen J, Malkia E. Neuromuscular function during therapeutic knee exercise under water and on dry land. Arch Phys Med Rehabil 2001; 82(10): 1446-52.

16. Prins JA, Cutner D. Aquatic therapy in the rehabilitation of athletic injuries. Clin Sports Med 1999; 18(2): 447-61.

17. Roos EM, Roos HP, Lohmander LS, Ekdahl C, Beynnon BD. Knee injury and osteoarthritis outcome score (KOOS) - development of a self-administered outcome measure. J Orthop Sports Phys Ther 1998; 28: 88-96.

18. Shelbourne KD, Nitz P. Accelerated rehabilitation after anterior cruciate ligament reconstruction. Am J Sports Med 1990; 18(3): 292-9.

19. Thalheimer W, Cook $S$. How to calculate effect sizes from published research articles: A simplified methodology. Retrieved 2008 from http:// work-learning.com/effect_sizes.htm.

20. Thein JM, Brody LT. Aquatic-based rehabilitation and training for the elite athlete. J Orthop Sports Phys Ther 1998; 27(1): 32-41.

21. Tovin BJ, Wolf SL, Greenfield BH, Crouse J, Woodfin BA. Comparison of the effects of exercises in water and on land on the rehabilitation of patients with intra-articular anterior cruciate ligament reconstructions. Phys Ther 2004; 74(8): 710-9. 